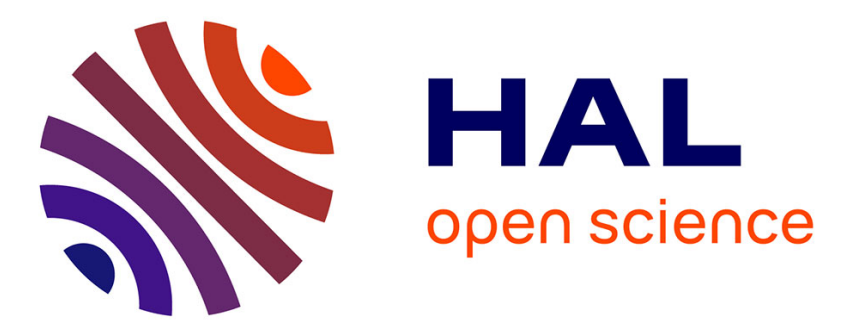

\title{
A crystal plasticity based approach for the modelling of high cycle fatigue damage in metallic materials
}

Jihed Zghal, Hela Gmati, Charles Mareau, Franck Morel

\section{To cite this version:}

Jihed Zghal, Hela Gmati, Charles Mareau, Franck Morel. A crystal plasticity based approach for the modelling of high cycle fatigue damage in metallic materials. International Journal of Damage Mechanics, 2016, 25 (5), pp.611-628. 10.1177/1056789516650247 . hal-01361077

\section{HAL Id: hal-01361077 \\ https://hal.science/hal-01361077}

Submitted on 6 Sep 2016

HAL is a multi-disciplinary open access archive for the deposit and dissemination of scientific research documents, whether they are published or not. The documents may come from teaching and research institutions in France or abroad, or from public or private research centers.
L'archive ouverte pluridisciplinaire HAL, est destinée au dépôt et à la diffusion de documents scientifiques de niveau recherche, publiés ou non, émanant des établissements d'enseignement et de recherche français ou étrangers, des laboratoires publics ou privés. 


\title{
A crystal plasticity based approach for the modelling of high cycle fatigue damage in metallic materials
}

\author{
Jihed Zghal', Hela Gmati², Charles Mareau \\ and Franck Morel $^{2}$
}

\begin{abstract}
In this paper, a polycrystalline model is proposed to describe the fatigue behaviour of metallic materials in the high cycle fatigue regime. The model is based on a multiscale approach, which allows the connection of local deformation and damage mechanisms to macroscopic behaviour. To consider the anisotropy of plastic properties, the constitutive model is developed at the grain scale within a crystal plasticity framework. A phenomenological approach, which requires the introduction of a damage variable for each slip system, is used to account for the anisotropic nature of damage. The constitutive model is then integrated within a self-consistent formulation to consider the polycrystalline nature of metallic materials. Finally, the proposed model is used to describe the high cycle fatigue behaviour of a medium carbon steel $(0.35 \% \mathrm{C})$. With a proper adjustment of material parameters, the model is capable of correctly reproducing fatigue test results, even for complex loading conditions (multiaxial, non-proportional). According to the model, damage is found to be highly localized in some specific grains. Also, while fatigue damage results in a progressive decrease in elastic stiffness at the crystal scale, the elastic properties are not significantly affected at the macroscopic scale. The model is used to study the correlation between energy dissipation and fatigue damage. According to the numerical results, no evident correlation between fatigue damage and energy dissipation is observed.
\end{abstract}

\section{Keywords}

Crystal plasticity, fatigue, damage

\footnotetext{
IIRT Jules Verne, France

${ }^{2}$ Arts et Métiers ParisTech, LAMPA, France
} 


\section{Introduction}

For metallic materials, fatigue behaviour in the high cycle fatigue regime is importantly influenced by microstructural heterogeneities (McDowell, 1996; Suresh, 1998). Indeed, for such materials, depending on the local microstructural features (e.g. crystallographic orientation, grain shape, defects), large fluctuations of the stress field are usually observed. As a result, although macroscopic behaviour is essentially elastic during high cycle fatigue loadings, it is possible for important plastic strains to accumulate locally, perhaps resulting in the nucleation of fatigue cracks. However, as noted by Przybyla et al. (2010), the investigation of the processes leading to the nucleation and propagation of fatigue cracks is complex because such phenomena involve different length scales, corresponding to different types of interaction with microstructural heterogeneities.

Computational methods, which allow the study of specific microstructural configurations, are a possible way towards a better understanding of the role of microstructure on the mechanisms governing crack initiation and crack propagation. Indeed, the development of polycrystalline models has offered some new possibilities for investigating and quantifying the role of microstructural heterogeneities. For instance, McDowell (2007) and Przybyla et al. (2010) have developed some microstructure-sensitive models for high cycle fatigue to include the influence of both loading conditions and microstructure variability. Bennett and McDowell (2003) and Repetto and Ortiz (1997) have used computational crystal plasticity methods to obtain statistical information concerning the dependence of the nucleation process regarding crystallographic orientation. The influence of internal length scales on fatigue crack initiation has been investigated with strain-gradient based plasticity models by Dunne et al. (2007) and Sweeney et al. (2013).

An alternative method of describing the influence of microstructure on high cycle fatigue behaviour is based on continuum damage mechanics. Continuum damage mechanics allows for consideration of the degradation of mechanical properties by explicitly introducing some damage variables in the constitutive relations (Lemaitre, 1996). However, although high cycle fatigue damage is characterized by a strong spatial localization, very few damage models were developed within the context of crystal plasticity. Monchiet et al. (2006) developed a damage model that assumes the initiation of microcracks along persistent slip bands to result from the production and the agglomeration of vacancies. The approach of Huyen et al. (2008) is based on the introduction of a damage variable for each slip system to account for the directional nature of damage. Nevertheless, although different numerical studies have emphasized the importance of elastic anisotropy in the crack nucleation process within the high cycle fatigue regime (Robert et al., 2012; Sauzay and Gilormini, 2002), this aspect is ignored in the aforementioned studies. Also, while the initial orientation of fatigue cracks is strongly dependent on the crystallographic orientation, the anisotropic nature of damage is often not considered.

In this work, a polycrystalline model is developed to describe the influence of microstructural heterogeneities on the high cycle fatigue behaviour of metallic materials. This model, whose development is based on continuum damage mechanics, aims at integrating (i) the anisotropic nature of damage and (ii) the influence of elastic anisotropy.

This paper is structured as follows. The first part is dedicated to the description of the polycrystalline model. The model is based on a multiscale approach that allows connection of local deformation and damage mechanisms to macroscopic behaviour. To consider the anisotropy of plastic properties, the constitutive model is developed at the grain scale within a crystal plasticity framework. A phenomenological approach, which is based on continuum damage mechanics, is used to introduce fatigue damage within the constitutive relations. Finally, to account for the polycrystalline nature of metallic materials, the constitutive model is incorporated within a self-consistent formulation. In the second part of this paper, the model is used to describe the high cycle fatigue behaviour 
of a medium carbon steel $(0.35 \% \mathrm{C})$. The studied material is briefly described and the experimental dataset, which was obtained by Flacelière et al. (2007a,b), Gadouini et al. (2008) and Vu (2009), is presented. The parameter identification procedure is then detailed. Finally, the model is used to investigate the spatial localization of fatigue damage, as well as the coupling of fatigue damage with both elasticity and plasticity.

\section{Model description}

\section{Local constitutive model}

Considering a single crystallite, the adoption of the infinitesimal strain theory allows the strain tensor $\varepsilon$ to be decomposed into elastic (superscript e) and plastic (superscript p) contributions

$$
\boldsymbol{\varepsilon}=\boldsymbol{\varepsilon}^{e}+\boldsymbol{\varepsilon}^{p}
$$

At each time $t$, the state of the crystallite can be defined with an appropriate set of state variables. If we restrict ourselves to isothermal processes, the infinitesimal strain tensor $\varepsilon$ is chosen as the sole observable state variable. The plastic strain tensor $\varepsilon^{p}$ is taken as an internal variable. Also, for a given slip system ( and the slip direction $\boldsymbol{m}^{s}$, some additional internal variables are introduced. The variables $q^{s}$ and $y^{s}$ are, respectively, associated with isotropic and kinematic hardening and the variable $d^{s}$ (with $\left.d^{s} \in[0,1]\right)$ corresponds to damage. The introduction of a damage variable for each slip system is a concept first proposed by Saanouni and Abdul-Latif (1996), and has been further developed by Abdul-Latif and Mounounga (2009), Chadli and Abdul-Latif (2005) and Mounounga et al. (2001).

The definition of the driving forces associated with the different state variables is obtained from a thermodynamic potential. In this work, the thermodynamic potential is given by the Helmholtz free energy density $\psi$, whose expression is

$$
\begin{aligned}
\rho \psi= & \frac{1}{2}\left(\boldsymbol{\varepsilon}-\boldsymbol{\varepsilon}^{p}\right): \boldsymbol{c}:\left(\boldsymbol{\varepsilon}-\boldsymbol{\varepsilon}^{p}\right) \\
& +\frac{1}{2} Q \sum_{s} q^{s} \sum_{t} H^{s t} q^{t}+\frac{1}{2} A \sum_{s} y^{s} y^{s}
\end{aligned}
$$

where $\rho$ is the mass density, $c$ is the elastic stiffness tensor, $Q$ and $A$ are the isotropic and kinematic hardening moduli, respectively, and $H$ is a $N \times N$ matrix, which is used to describe self and latent isotropic hardening. The anisotropic nature of damage is captured through the dependence of the elastic stiffness tensor $c$ regarding the damage variables. To evaluate how elastic properties (i.e. the capacity to transmit internal forces) are impacted by damage, a damage tensor $\boldsymbol{d}^{s}$ of order 4 is introduced for each slip system $s$. The damage tensor $\boldsymbol{d}^{s}$ is defined as a function of the scalar damage variable $d^{s}$ according to

$$
\begin{aligned}
\boldsymbol{d}^{s}= & h^{s} \boldsymbol{n}^{s} \otimes \boldsymbol{n}^{s} \otimes \boldsymbol{n}^{s} \otimes \boldsymbol{n}^{s}+\frac{1}{2} d^{s} Z\left(\boldsymbol{n}^{s} \otimes \boldsymbol{m}^{s}+\boldsymbol{m}^{s} \otimes \boldsymbol{n}^{s}\right) \otimes\left(\boldsymbol{n}^{s} \otimes \boldsymbol{m}^{s}+\boldsymbol{m}^{s} \otimes \boldsymbol{n}^{s}\right) \\
& +\frac{1}{2} d^{s} Z\left(\boldsymbol{n}^{s} \otimes \boldsymbol{p}^{s}+\boldsymbol{p}^{s} \otimes \boldsymbol{n}^{s}\right) \otimes\left(\boldsymbol{n}^{s} \otimes \boldsymbol{p}^{s}+\boldsymbol{p}^{s} \otimes \boldsymbol{n}^{s}\right)
\end{aligned}
$$


where $\boldsymbol{p}^{s}$ is a unit vector such that $\boldsymbol{p}^{s}=\boldsymbol{n}^{s} \times \boldsymbol{m}^{s}, Z$ is a material parameter describing the shear stress sensitivity and $h^{s}$ is a function accounting for closure effects

$$
h^{s}= \begin{cases}d^{s} & \text { if } \boldsymbol{n}^{s} \cdot \boldsymbol{\varepsilon} \cdot \boldsymbol{n}^{s} \geq 0 \\ 0 & \text { if } \boldsymbol{n}^{s} \cdot \boldsymbol{\varepsilon} \cdot \boldsymbol{n}^{s}<0\end{cases}
$$

The elastic stiffness tensor $c$ is then calculated as a function of the damage tensors according to

$$
c=\left(1-\sum_{s} d^{s}\right): \tilde{c}:\left(1-\sum_{s} d^{s}\right)
$$

where $\tilde{\boldsymbol{c}}$ is the initial elastic stiffness tensor (corresponding to an undamaged configuration) and $\mathbf{1}$ is the fourth-rank identity tensor. According to this definition, the elastic stiffness tensor is necessarily symmetrical and positive definite. Also, this formulation assumes that, for a damaged system, both the Young modulus and the shear modulus are reduced in some specific directions, depending on the slip system orientations. The proposed approach is thus designed to describe the nucleation and propagation of fatigue cracks along persistent slip bands. It would be inappropriate for some other initiation mechanisms.

The driving forces associated with the different state variables are given by the derivatives of the thermodynamic potential $\psi$ regarding the corresponding state variables

$$
\begin{gathered}
\boldsymbol{\sigma}=\rho \frac{\partial \psi}{\partial \boldsymbol{\varepsilon}}=\boldsymbol{c}:\left(\boldsymbol{\varepsilon}-\boldsymbol{\varepsilon}^{p}\right) \\
-\boldsymbol{\sigma}=\rho \frac{\partial \psi}{\partial \boldsymbol{\varepsilon}^{p}}=-\boldsymbol{c}:\left(\boldsymbol{\varepsilon}-\boldsymbol{\varepsilon}^{p}\right) \\
r^{s}=\rho \frac{\partial \psi}{\partial q^{s}}=Q \sum_{t} H^{s t} q^{t} \\
x^{s}=\rho \frac{\partial \psi}{\partial y^{s}}=A y^{s} \\
-f^{s}=\rho \frac{\partial \psi}{\partial d^{s}}=\frac{1}{2}\left(\boldsymbol{\varepsilon}-\boldsymbol{\varepsilon}^{p}\right): \frac{\partial \boldsymbol{c}}{\partial d^{s}}:\left(\boldsymbol{\varepsilon}-\boldsymbol{\varepsilon}^{p}\right)
\end{gathered}
$$

where $\boldsymbol{\sigma}$ is the stress tensor, $r^{s}$ and $x^{s}$ are the shear stresses associated, respectively, with isotropic and kinematic hardening and $-f^{s}$ is the driving force for damage. It is worth noting that, following the approach of Lemaitre (1996), the driving force for damage corresponds to the elastic strain energy release rate.

To consider how damage affects mechanical behaviour, a common strategy consists of adopting a strain equivalence approach to define the effective stress state (Lemaitre, 1996). In this work, an alternative method, which is based on a free energy equivalence principle (Cordebois and Sidoroff, 1979; Saanouni and Abdul-Latif, 1996), is employed. According to this method, an effective strain tensor $\tilde{\boldsymbol{\varepsilon}}$ and an effective plastic strain tensor $\tilde{\boldsymbol{\varepsilon}}^{p}$ are introduced to define an equivalent undamaged 
crystal, for which the free energy density is the same as the damaged crystal that has been considered so far

$$
\rho \psi=\frac{1}{2}\left(\tilde{\boldsymbol{\varepsilon}}-\tilde{\boldsymbol{\varepsilon}}^{p}\right): \tilde{\boldsymbol{c}}:\left(\tilde{\boldsymbol{\varepsilon}}-\tilde{\boldsymbol{\varepsilon}}^{p}\right)+\frac{1}{2} Q \sum_{s} q^{s} \sum_{t} H^{s t} q^{t}+\frac{1}{2} A \sum_{s} y^{s} y^{s}
$$

For equations (2) and (11) to be equivalent, the effective total and plastic strain tensors must satisfy the following equalities

$$
\begin{gathered}
\tilde{\boldsymbol{\varepsilon}}=\left(1-\sum_{S} \boldsymbol{d}^{S}\right): \boldsymbol{\varepsilon} \\
\tilde{\boldsymbol{\varepsilon}}^{p}=\left(1-\sum_{S} \boldsymbol{d}^{S}\right): \boldsymbol{\varepsilon}^{p}
\end{gathered}
$$

The effective stress tensor $\tilde{\boldsymbol{\sigma}}$ is then given by:

$$
\tilde{\boldsymbol{\sigma}}=\rho \frac{\partial \psi}{\partial \tilde{\boldsymbol{\varepsilon}}}=-\rho \frac{\partial \psi}{\partial \tilde{\boldsymbol{\varepsilon}}^{p}}=\left(\mathbf{1}-\sum_{s} \boldsymbol{d}^{S}\right)^{-1}: \boldsymbol{\sigma}
$$

The effective stress state can be used to establish the evolution laws associated with the different internal variables. For an undamaged crystal, the plastic deformation process results from a shear mechanism. Within a crystal plasticity framework, it is therefore possible to express the effective plastic strain rate as a function of the effective shear rates $\dot{\tilde{\gamma}}^{s}$ associated with the different slip systems

$$
\dot{\tilde{\boldsymbol{\varepsilon}}}^{p}=\sum_{s} \boldsymbol{l}^{s} \dot{\tilde{\gamma}}^{s}-\left(\sum_{s} \dot{\boldsymbol{d}}^{s}\right): \boldsymbol{\varepsilon}^{p}
$$

where $\boldsymbol{l}^{s}$ is the symmetric part of the Schmid tensor

$$
\boldsymbol{l}^{s}=\frac{1}{2}\left(\boldsymbol{n}^{s} \otimes \boldsymbol{m}^{s}+\boldsymbol{m}^{s} \otimes \boldsymbol{n}^{s}\right)
$$

The effective plastic shear rate $\dot{\tilde{\gamma}}^{s}$ associated with the slip system $s$ is connected to the corresponding effective resolved shear stress $\tilde{\tau}^{s}$, the kinematic shear stress $x^{s}$ and the isotropic stress $r^{s}$, according to the viscoplastic flow rule proposed by Méric and Cailletaud (1991)

$$
\dot{\tilde{\gamma}}^{s}=\left(\frac{\left\langle\left|\tilde{\tau}^{s}-x^{s}\right|-r^{s}-R_{0}\right\rangle}{K}\right)^{N} \operatorname{sign}\left(\tilde{\tau}^{s}-\tilde{x}^{s}\right)
$$

where $K$ is a viscosity parameter, $N$ is the strain rate sensitivity parameter, $R_{0}$ is the initial value of the critical shear stress and $\tilde{\tau}^{s}$ is the effective resolved shear stress

$$
\tilde{\tau}^{s}=\boldsymbol{m}^{s} \cdot \tilde{\boldsymbol{\sigma}} \cdot \boldsymbol{n}^{s}
$$


The evolution of the isotropic and kinematic hardening variables is given by the following non-linear rules

$$
\begin{aligned}
& \dot{q}^{s}=\left|\dot{\tilde{\gamma}}^{s}\right|-B q^{s}\left|\dot{\tilde{\gamma}}^{s}\right| \\
& \dot{y}^{s}=\dot{\tilde{\gamma}}^{s}-D y^{s}\left|\dot{\tilde{\gamma}}^{s}\right|
\end{aligned}
$$

where $B$ and $D$ are material parameters. Such hardening rules are well suited for materials exhibiting a stabilized cyclic behaviour. They would have to be modified for other types of cyclic behaviour. The evolution of damage is described according to a rate-dependent type of relation

$$
\dot{d}^{s}=W\left\langle\beta^{S}\right\rangle^{M}\left|\dot{\tilde{\gamma}}^{S}\right|^{P}
$$

This relation, for which $W, M$ and $P$ are material parameters, assumes a strong coupling between damage and plasticity, since damage develops only in the presence of a plastic activity. Indeed, following the idea of Lemaitre and Chaboche (1994), damage is governed by the most dissipative mechanism, which, in this work, is plastic slip. Considering the damaged crystal, the actual plastic strain rate tensor is obtained by combining equations (13) and (15)

$$
\dot{\boldsymbol{\varepsilon}}^{p}=\left(\mathbf{1}-\sum_{s} \boldsymbol{d}^{s}\right)^{-1}:\left(\dot{\tilde{\boldsymbol{\varepsilon}}}^{p}+\sum_{s} \dot{\boldsymbol{d}}^{s}: \boldsymbol{\varepsilon}^{p}\right)
$$

Since the constitutive model does not fall into the category of generalized standard models, the positivity of the intrinsic dissipation source must be verified. The intrinsic dissipation source $d_{1}=\boldsymbol{\sigma}: \dot{\boldsymbol{\varepsilon}}-\dot{\psi}$ is given by

$$
d_{1}=\underbrace{\sum_{s} f^{s} \dot{d}^{s}}_{\text {damage }}+\underbrace{\sum_{s} \tilde{\tau}^{s} \dot{\tilde{\gamma}}^{s}-\sum_{s} r^{s} \dot{q}^{s}-\sum_{s} x^{s} \dot{y}^{s}}_{\text {plasticity }}
$$

With the evolution equations (15) to (21), the intrinsic dissipation source is necessarily positive. According to the expression of the dissipation source, for a given slip system, both plasticity and damage contribute to heat dissipation. Also, the plastic work, whose rate is given by $\tilde{\tau}^{s} \dot{\tilde{\gamma}}^{s}$, is not entirely dissipated as heat. Indeed, some elastic strain energy is stored within the material, mostly because of the internal stresses associated with metallurgical defects (e.g. dislocations, vacancies). Since kinematic hardening and isotropic hardening are governed by the multiplication and organization of metallurgical defects, the energy storage rate is represented with the terms $r^{s} \dot{q}^{s}$ and $x^{s} \dot{y}^{s}$.

\section{Self-consistent model}

Considering a polycrystalline material for which it is possible to define a representative volume element $V$, it is now necessary to integrate the constitutive model within a multiscale framework. Since polycrystalline aggregates can be conceived as a disordered collection of crystallites that are perfectly bonded to each other, the self-consistent method is appropriate for computing the effective behaviour. In this work, the self-consistent model proposed by Mareau and Berbenni (2015), which is briefly described here, is used. As shown in Robert and Mareau (2015), this model provides reasonable estimates of the effective behaviour of polycrystalline aggregates within short computation times. 
Within the infinitesimal strain framework, the strain rate tensor associated with a given crystallite is decomposed into elastic and plastic contributions

$$
\dot{\boldsymbol{\varepsilon}}=\dot{\boldsymbol{\varepsilon}}^{e}+\dot{\boldsymbol{\varepsilon}}^{p}
$$

To apply the self-consistent method, the elastic and plastic constitutive relations are rewritten in the following affine form

$$
\begin{gathered}
\dot{\boldsymbol{\varepsilon}}^{e}=\boldsymbol{s}: \dot{\boldsymbol{\sigma}}+\dot{\alpha} \\
\dot{\boldsymbol{\varepsilon}}^{p}=\boldsymbol{m}: \boldsymbol{\sigma}+\dot{\boldsymbol{\eta}}
\end{gathered}
$$

where $\boldsymbol{s}\left(=\boldsymbol{c}^{-1}\right)$ and $\boldsymbol{m}\left(=\boldsymbol{b}^{-1}\right)$ are the tangent elastic and viscoplastic compliances tensors, respectively, and $\dot{\boldsymbol{\alpha}}$ and $\dot{\boldsymbol{\eta}}$ are the back-extrapolated elastic and viscoplastic strain rate tensors, respectively. In these relations, the presence of the back-extrapolated elastic and viscoplastic strain rates results from the non-linear character of the elastic and plastic constitutive relations. The viscoplastic compliances tensor is obtained from a first-order Taylor expansion of the flow rule

$$
\boldsymbol{m}=\frac{\partial \dot{\boldsymbol{\varepsilon}}^{p}}{\partial \boldsymbol{\sigma}}=\left(\mathbf{1}-\sum_{s} \boldsymbol{d}^{s}\right)^{-1}: \tilde{\boldsymbol{m}}:\left(\mathbf{1}-\sum_{s} \boldsymbol{d}^{s}\right)^{-1}
$$

with

$$
\tilde{\boldsymbol{m}}=\left(\sum_{s} \frac{\partial \dot{\tilde{\gamma}}^{s}}{\partial \tilde{\tau}^{s}} \boldsymbol{l}^{s} \otimes \boldsymbol{l}^{s}\right)
$$

The back-extrapolated strain rate tensors $\dot{\alpha}$ and $\dot{\eta}$ are given by

$$
\begin{gathered}
\dot{\boldsymbol{\alpha}}=\dot{\boldsymbol{\varepsilon}}^{e}-\boldsymbol{s}: \dot{\boldsymbol{\sigma}} \\
\dot{\boldsymbol{\eta}}=\dot{\boldsymbol{\varepsilon}}^{p}-\boldsymbol{m}: \boldsymbol{\sigma}
\end{gathered}
$$

The approach of Mareau and Berbenni (2015) involves first finding, independently, the effective elastic properties and the effective viscoplastic properties. Indeed, treating crystallites as ellipsoidal inclusions and temporarily considering a purely elastic behaviour (i.e. $\dot{\boldsymbol{\varepsilon}}=\dot{\boldsymbol{\varepsilon}}^{e}$ ), the application of the self-consistent method enables calculation of the effective elastic compliance tensor $\boldsymbol{S}=\boldsymbol{C}^{-1}$ and the effective back-extrapolated elastic strain rate tensor $\dot{A}$. For the purely elastic problem, the effective properties $\boldsymbol{C}$ and $\dot{\boldsymbol{A}}$ are given by

$$
\begin{gathered}
C=\overline{c: a^{c}} \\
\dot{A}=\overline{\boldsymbol{S}: \boldsymbol{a}^{C^{T}}: c: \dot{\alpha}}
\end{gathered}
$$

where $\bar{x}$ denotes the average of the quantity $x$ over the representative volume element. The elastic strain localization tensor $\boldsymbol{a}^{C}$ is computed according to

$$
a^{C}=\left(1+\Gamma^{C}:(c-C)\right)^{-1}
$$


The modified Green operator $\Gamma^{C}$ associated with the equivalent elastic homogeneous medium is determined quite easily from the solution of an Eshelby inclusion problem (Eshelby, 1957). In a very similar fashion, when a purely viscoplastic behaviour is considered (i.e. $\dot{\boldsymbol{\varepsilon}}=\dot{\boldsymbol{\varepsilon}}^{p}$ ), the effective viscoplastic compliance tensor $\boldsymbol{M}=\boldsymbol{B}^{-1}$ and the effective back-extrapolated viscoplastic strain rate tensor $\dot{N}$ can be determined with the self-consistent method

$$
\begin{gathered}
\boldsymbol{B}=\overline{\boldsymbol{b}: \boldsymbol{a}^{B}} \\
\dot{\boldsymbol{N}}=\overline{\boldsymbol{S}: \boldsymbol{a}^{B^{T}}: \boldsymbol{b}: \dot{\boldsymbol{\eta}}}
\end{gathered}
$$

The viscoplastic strain localization tensor $\boldsymbol{a}^{B}$ is obtained by

$$
\boldsymbol{a}^{B}=\left(\mathbf{1}+\boldsymbol{\Gamma}^{B}:(\boldsymbol{b}-\boldsymbol{B})\right)^{-1}
$$

where $\Gamma^{B}$ is the modified Green operator associated with the equivalent viscoplastic homogeneous medium. According to the formulation of Mareau and Berbenni (2015), the self-consistent solutions obtained for the elastic and viscoplastic heterogeneous problems can be combined to solve the complete (i.e. elastic-viscoplastic) heterogeneous problem. This strategy allows for computation of the average strain rate tensor for each crystallite with the following localization rule

$$
\begin{aligned}
\dot{\boldsymbol{\varepsilon}}=\dot{\boldsymbol{E}} & +\boldsymbol{\Gamma}^{C}:(\boldsymbol{c}: \dot{\boldsymbol{\alpha}}-\boldsymbol{C}: \dot{\boldsymbol{A}})+\boldsymbol{\Gamma}^{B}:(\boldsymbol{b}: \dot{\boldsymbol{\eta}}-\boldsymbol{B}: \dot{\boldsymbol{N}})+\boldsymbol{\Gamma}^{C}:\left((\boldsymbol{C}-\boldsymbol{c}): \dot{\boldsymbol{\varepsilon}}^{e}+\boldsymbol{c}: \boldsymbol{a}^{C}:\left(\boldsymbol{S}: \dot{\boldsymbol{\Sigma}}+\dot{\boldsymbol{A}}-\overline{\dot{\boldsymbol{\varepsilon}}}^{e}\right)\right) \\
& +\boldsymbol{\Gamma}^{B}:\left((\boldsymbol{B}-\boldsymbol{b}): \dot{\boldsymbol{\varepsilon}}^{p}+\boldsymbol{b}: \boldsymbol{a}^{B}:\left(\boldsymbol{M}: \boldsymbol{\Sigma}+\dot{\boldsymbol{N}}-\overline{\boldsymbol{\varepsilon}}^{p}\right)\right)+\left(\boldsymbol{\Gamma}^{C}: \boldsymbol{C}-\boldsymbol{\Gamma}^{B}: \boldsymbol{B}\right):\left(\dot{\boldsymbol{\varepsilon}}^{p}-\boldsymbol{a}^{B}: \overline{\dot{\boldsymbol{\varepsilon}}}^{p}\right) \\
& -\left(\boldsymbol{\Gamma}^{C}: \boldsymbol{C}-\boldsymbol{\Gamma}^{B}: \boldsymbol{B}\right):\left(\boldsymbol{a}^{B}: \boldsymbol{\Gamma}^{B}:(\boldsymbol{b}: \dot{\boldsymbol{\eta}}-\boldsymbol{B}: \dot{\boldsymbol{N}})\right)
\end{aligned}
$$

where $\boldsymbol{E}$ is the prescribed macroscopic strain tensor and $\boldsymbol{\Sigma}$ is the macroscopic stress tensor. The effective response of the representative volume element is given by the macroscopic stress rate $\dot{\boldsymbol{\Sigma}}$, which is obtained by combining the classical averaging relation of homogenization theory $\dot{\boldsymbol{\Sigma}}=\overline{\dot{\boldsymbol{\sigma}}}$ with the constitutive relation, equation (25), and the localization rule, equation (37).

Because of the limitations of the self-consistent approximation, the proposed model is developed for materials for which fatigue damage is essentially an intragranular process. Indeed, the selfconsistent formulation underestimates the influence of neighbouring grains (Robert and Mareau, 2015), so internal stresses near interfaces are not accurately evaluated with this formulation. The application of the model to materials for which the fatigue behaviour is significantly impacted by grain or twin boundaries would therefore be inappropriate.

\section{Application to a medium carbon steel}

\section{Material description}

To test the validity of the proposed model, the fatigue behaviour of a medium carbon steel (SAE 1035) was studied. The experimental results used for the present application were extracted from the work of Flacelière et al. (2007a,b), Gadouini et al. (2008) and Vu (2009). The chemical composition is given in Table 1 and the mechanical properties, obtained for quasistatic uniaxial loading conditions, are listed in Table 2. The material is two-phased (ferrite and cementite), with a volume fraction of proeutectoid ferrite of about $55 \%$ and a volume fraction of pearlite of about $45 \%$. 
Table I. Chemical composition (weight concentration) of SAE 1035 steel (Vu, 2009).

\begin{tabular}{llllll}
\hline $\mathrm{C}$ & $\mathrm{Si}$ & $\mathrm{Mn}$ & $\mathrm{S}$ & $\mathrm{P}$ & $\mathrm{Ni}$ \\
$0.36 \%$ & $0.27 \%$ & $0.6 \%$ & $0.009 \%$ & $0.034 \%$ & $0.07 \%$ \\
$\mathrm{Cr}$ & $\mathrm{Co}$ & $\mathrm{Mo}$ & $\mathrm{W}$ & $\mathrm{V}$ & $\mathrm{Ti}$ \\
$0.14 \%$ & $0.07 \%$ & $0.01 \%$ & $0.01 \%$ & $0.005 \%$ & $0.009 \%$ \\
\hline
\end{tabular}

Table 2. Mechanical properties of SAE 1035 steel (Gadouini et al., 2008).

\begin{tabular}{lr}
\hline Young modulus & $195 \mathrm{GPa}$ \\
Yield strength & $359 \mathrm{MPa}$ \\
Ultimate strength & $594 \mathrm{MPa}$ \\
Elongation at break & $47 \%$ \\
\hline
\end{tabular}

The microstructure consists of equiaxed grains with an average size of approximately $20 \mu \mathrm{m}$ for ferrite and $15 \mu \mathrm{m}$ for pearlite.

In this work, the simulations were performed with a polycrystalline aggregate consisting of 1000 spherical grains with random crystallographic orientations. The behaviour of ferritic grains is described with the constitutive model presented in the first part of this paper. Both the $\{110\}\langle 111\rangle$ and $\{112\}\langle 111\rangle$ slip systems were considered for the viscoplastic behaviour of the ferritic phase. For pearlitic grains, a specific treatment was adopted. Indeed, because pearlite is a lamellar aggregate of cementite (superscript $c$, volume fraction 12\%) and eutectoid ferrite (superscript $\alpha$, volume fraction $88 \%$ ), it is necessary to partition the strain tensor between both phases. In this work, an upper bound estimation, which assumes an uniform strain state within pearlitic grains, was used

$$
\varepsilon^{c}=\varepsilon^{\alpha}=\varepsilon
$$

A purely elastic behaviour was assumed for both eutectoid ferrite and cementite. The stress tensor associated with pearlitic grains is thus obtained with the averaging relation

$$
\boldsymbol{\sigma}=0.88 \boldsymbol{\sigma}^{\alpha}+0.12 \boldsymbol{\sigma}^{c}=\left(0.88 \boldsymbol{c}^{\alpha}+0.12 \boldsymbol{c}^{c}\right): \boldsymbol{\varepsilon}
$$

\section{Experimental data}

To determine the elastic-plastic cyclic behaviour, Gadouini et al. (2008) conducted strain-controlled cyclic tests with different strain amplitudes $(0.21 \%, 0.31 \%$ and $0.45 \%)$. (Gadouini et al. (2008) also performed a strain-controlled cyclic test with an amplitude of $1.2 \%$. The results of this test are not presented here as they were not considered for parameter identification.) For the different strain amplitudes, a stabilized behaviour was usually observed after 100 cycles. The associated cyclic stress-strain curves are plotted in Figure 1. Also, when the transient behaviour is observed (see Figure 2), peak stresses are found to decrease with an increasing number of cycles. The material therefore exhibits some cyclic softening. 


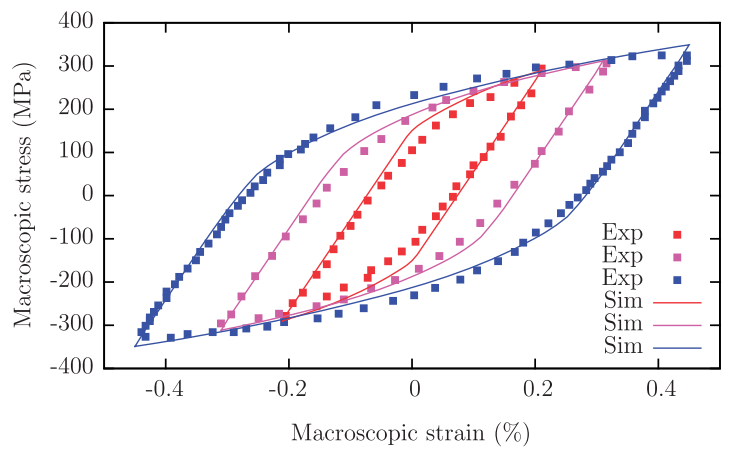

Figure I. Macroscopic stress-strain curves obtained for different strain-controlled cyclic tests with different strain amplitudes within the stabilized regime.

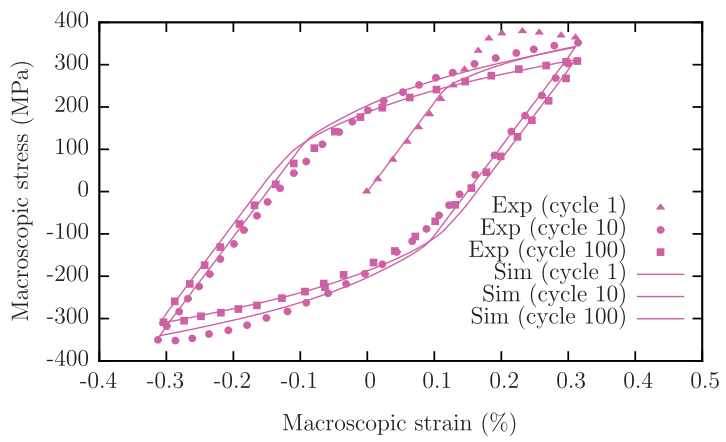

Figure 2. Macroscopic stress-strain curves obtained for a strain-controlled cyclic test with a strain amplitude of $0.31 \%$.

The fatigue strength of the SAE 1035 steel was evaluated by Flacelière et al. $(2007 a, b)$ and $\mathrm{Vu}$ (2009) from stress-controlled fatigue tests combining normal $\left(\Sigma_{11}\right)$ and shear $\left(\Sigma_{12}\right)$ stresses. The different tests were performed with sinusoidal waveforms and zero mean stresses. Each fatigue test is thus characterized with a normal stress amplitude $\Sigma_{11}^{a}$, a shear stress amplitude $\Sigma_{12}^{a}$, a pulsation $\omega$ and a phase angle $\delta$

$$
\begin{gathered}
\Sigma_{11}=\Sigma_{11}^{a} \sin (\omega t) \\
\Sigma_{12}=\Sigma_{12}^{a} \sin (\omega t+\delta)
\end{gathered}
$$

The introduction of the ratio $k=\Sigma_{12}^{a} / \Sigma_{11}^{a}$ allows for the distinction of five configurations: $k=0$ (tension or compression), $k=\infty$ (torsion), $k=0.5 / \delta=0^{\circ}$ (in-phase tension or torsion), $k=1 / \delta=0^{\circ}$ (in-phase tension or torsion) and $k=0.5 / \delta=90^{\circ}$ (out-of-phase tension or torsion). The experimental $S-N$ curves obtained for the different configurations are plotted in Figure 3. The stress amplitude is given in the form of a von Mises equivalent stress amplitude $\Sigma_{e q}^{a}$ whose definition is

$$
\Sigma_{e q}^{a}=\max _{t}\left(\sqrt{\Sigma_{11}^{2}(t)+3 \Sigma_{12}^{2}(t)}\right)
$$




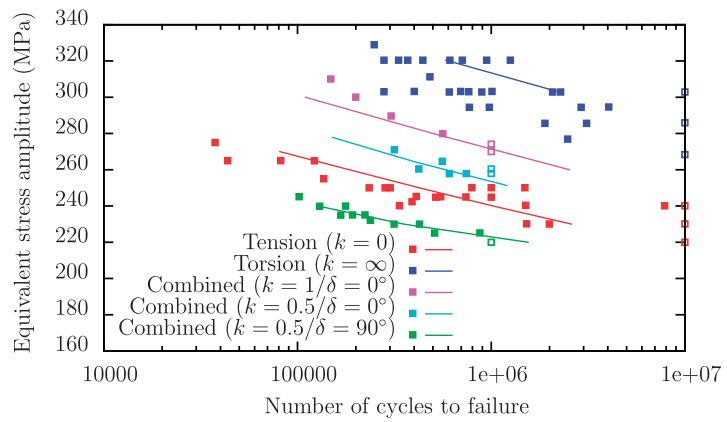

Figure 3. Evolution of the equivalent stress amplitude as a function of the number of cycles to failure for the different loading configurations. Numerical results are presented with solid lines, experimental results are presented with symbols.

\section{Parameter identification}

To identify the material parameters for the SAE 1035 steel, the following strategy was adopted. First, single-crystal elastic constants were taken from Sakata et al. (1989) for ferrite and from Ledbetter (2010) for cementite. Then, for ferrite, the parameters associated with the viscoplastic flow rule $\left(R_{0}, K\right.$ and $\left.N\right)$, the isotropic hardening rule $(Q, B$ and $H)$ and the kinematic hardening rule ( $A$ and $D$ ) were determined to match the experimental stress-strain curves obtained from straincontrolled cyclic tests. In this case, the interaction matrix $H$ is of size $N=24$. It is constructed from two coefficients: one for self-hardening and one for latent hardening. As shown in Figures 1 and 2, apart from the first cycle, the constitutive model provides a correct description of the cyclic behaviour. Finally, the material parameters relative to damage $(Z, W, M$ and $P$ ) were adjusted to reproduce the experimental $S-N$ curves. The material parameters are given in Table 3 . The fracture criterion used for computing the number of cycles to failure is based on the observation of the maximal value of the damage variable. More specifically, if the damage variable reaches a critical value (denoted $d^{c}$ ) for at least one slip system of one crystallite then the polycrystalline aggregate is considered to be fractured. The fracture criterion therefore reads

$$
\max _{V}\left(\max _{s}\left(d^{s}\right)\right)=d^{c}
$$

In this work, a value of 1 has been used for $d^{c}$. According to Figure 3, the agreement between numerical and experimental results is correct for the different loading conditions. Also, the proposed polycrystalline model enables calculation of the fatigue strength at $10^{6}$ cycles for the different loading configurations. As shown in Figure 4, when compared with the estimates obtained from some common fatigue criteria (Crossland, 1956; Dang Van, 1973), only the present model is able to account for the influence of non-proportionality. The present model thus seems capable of correctly describing the influence of loading conditions with a limited number of damage-related parameters.

\section{Results}

The proposed model allows for determination not only of the macroscopic behaviour but also the evolution of the local state. Indeed, it is possible to observe the evolution of the different state 
Table 3. Material parameters for SAE 1035 steel.

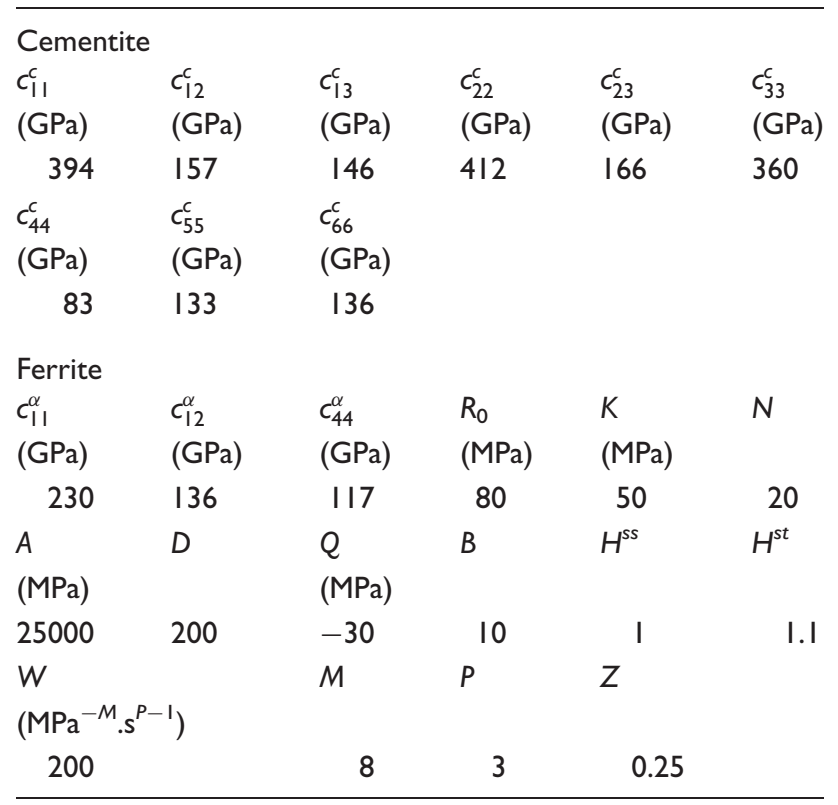

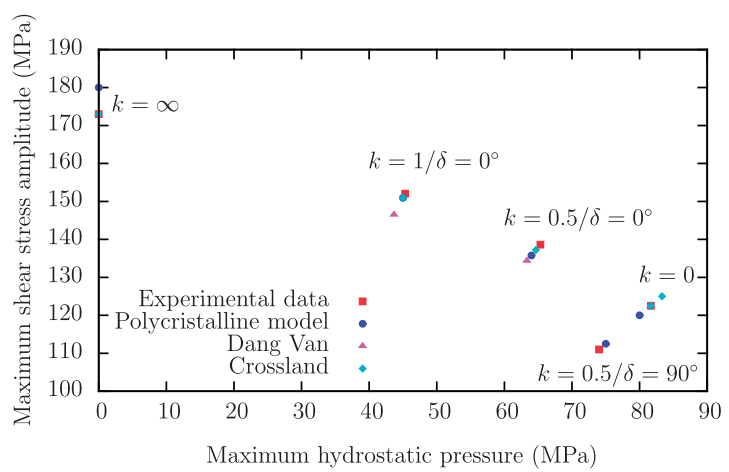

Figure 4. Evolution of the fatigue strength at $10^{6}$ cycles for the different loading configurations.

variables associated with a specific crystallite to better understand the development of damage, depending on the loading conditions.

Figure 5 shows the evolution of the damage variable associated with the critical system (i.e. $\max _{V}$ $\left.\left(\max _{s}\left(d^{S}\right)\right)\right)$ for different loading configurations. Independently of the loading conditions, the same type of evolution is obtained. A slow accumulation of damage is observed during the first stage of cyclic loading, while the final fracture is preceded by an important acceleration of the damage process.

To better understand the coupling between damage and plasticity, the damage variable $d^{s}$, obtained for each slip system of the polycrystalline aggregate, is plotted as a function of the 


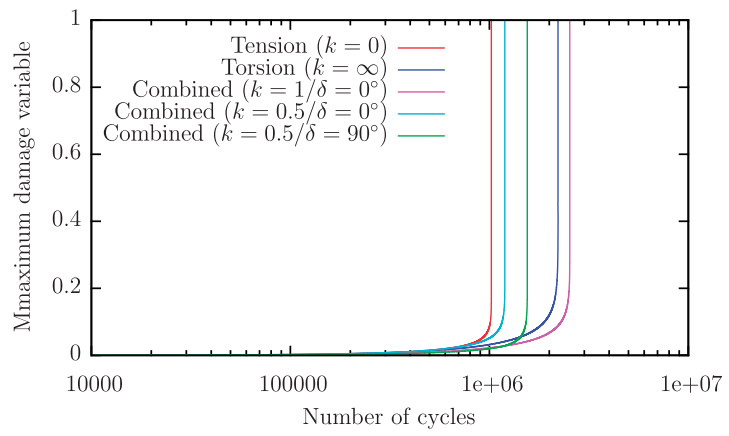

Figure 5. Evolutions of the damage variables associated with the critical system as a function of the number of cycles for the different loading configurations.

corresponding plastic shear strain variable $\left|\tilde{\gamma}^{s}\right|$ in Figure 6. The damage and plastic shear strain variables have been calculated at the end of the 10,000th cycle for the different loading configurations. For each loading configuration, the stress amplitudes $\Sigma_{11}^{a}$ and $\Sigma_{12}^{a}$ correspond to a number of cycles to failure $N_{f}$ of approximately $1 \times 10^{6}$. As shown in Figure 6, independently of the loading mode, damage is found to be highly localized in some specific systems. Also, although damage is generally important for slip systems with an important plastic activity, the critical system, for which the damage variable is maximal, does not display the highest plastic shear strain. Indeed, while plastic slip is governed by shear stresses only, the evolution of damage for a given slip system depends on both shear and normal stresses. As a result, the orientation of the critical system is strongly influenced by the competition between shear and normal stresses.

The impact of fatigue damage on elastic properties is evaluated by plotting the evolution of both the macroscopic and local Young moduli along the axial loading direction (direction 1) for the different loading configurations. To illustrate the influence of the unilateral damage condition, the minimum (upon tension) and maximum (upon compression) values of the elastic moduli are given for each loading cycle. The evolution of the macroscopic Young modulus is plotted in Figure 7(a). Independently of the loading configuration, no significant degradation of macroscopic elastic properties is observed because fatigue damage is highly localized in some specific crystallites. The evolution of the local Young modulus, which is calculated for the crystal containing the most critical system, is presented in Figure 7(b). Except from torsion, a progressive decrease of the Young modulus is observed for the different loading configurations. For the specific case of torsion, the local Young modulus is not significantly affected because of the anisotropic nature of damage. Indeed, for this loading configuration, the absence of normal stress strongly limits the impact of damage along the axial direction. Also, as shown by the compression and tension moduli, the introduction of an unilateral damage condition renders the elastic behaviour asymmetrical at the local scale. At the macroscopic scale, no major difference between tension and compression moduli is observed.

To better understand how the dissipative behaviour is affected by damage, the contributions of plasticity and damage to the macroscopic dissipation source $D_{1}$ were calculated. The macroscopic dissipation source $D_{1}$ is given by the volume average of the local dissipation source $d_{1}$

$$
D_{1}=\overline{d_{1}}
$$



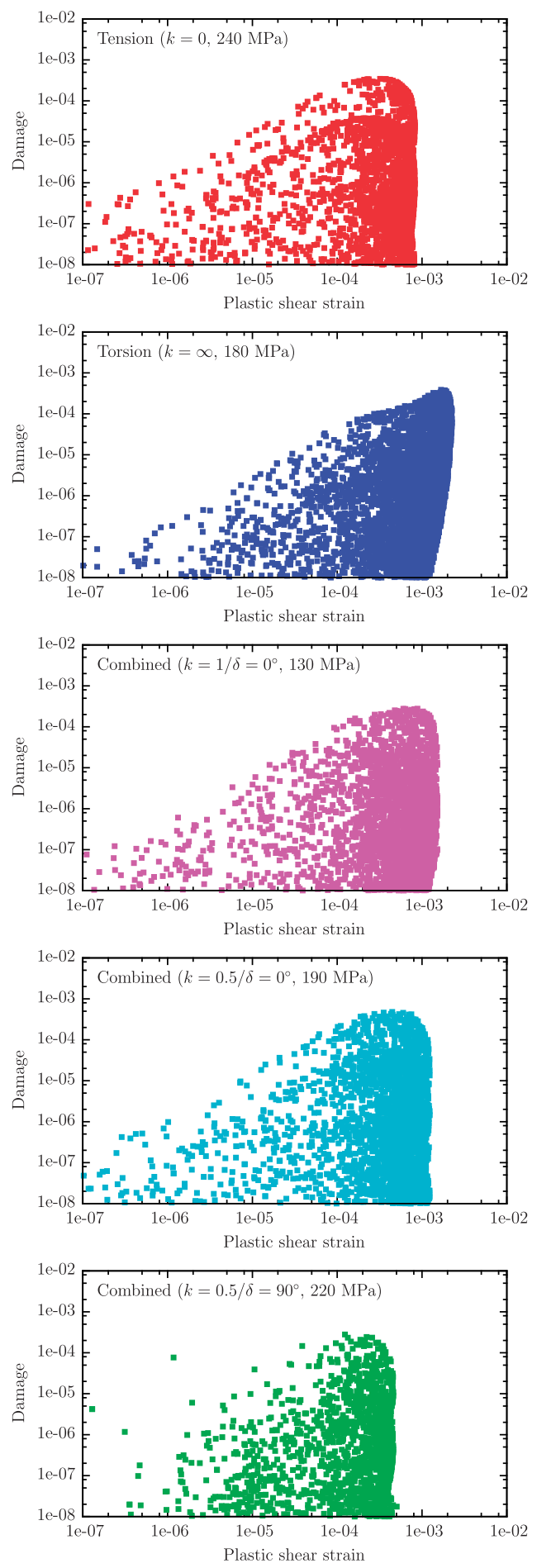

Figure 6. Representation of damage variables associated with the different systems as a function of the corresponding plastic shear strain variables for the different loading configurations. 
(a)

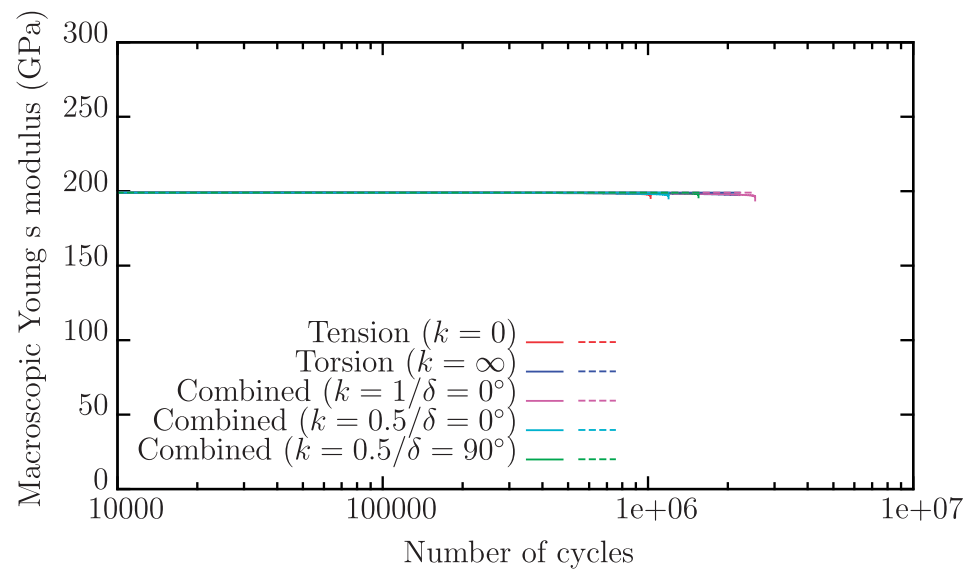

(b)

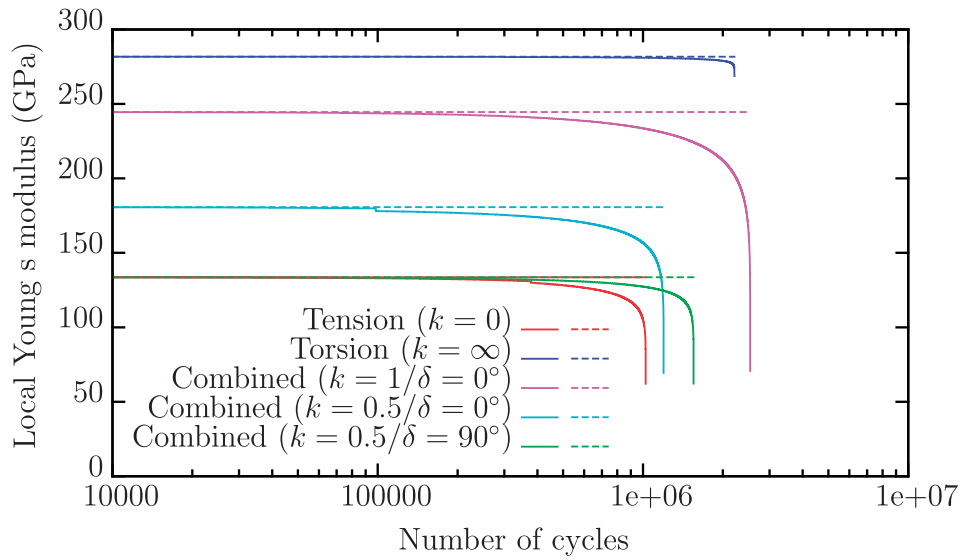

Figure 7. Evolution of (a) macroscopic and (b) local Young moduli along the axial direction as a function of the number of cycles for different loading configurations. The local Young modulus is calculated for the most damaged crystal. Solid lines correspond to tension moduli, dashed lines correspond to compression moduli.

For a given loading cycle, the average heat-dissipated energy per cycle is obtained by integrating the macroscopic dissipation source $D_{1}$ over one cycle period. To determine which mechanism is predominant, the evolution of the relative contribution of damage to the average heat-dissipated energy per cycle as a function of the number of cycles is plotted in Figure 8 for the different loading configurations. For any loading configuration, and even when the damage rate is important (i.e. prior to the final failure), the contribution associated with damage is negligible in comparison with the contribution associated with plasticity.

Moreover, various previous studies have mentioned a possible relation between high cycle fatigue properties and energy dissipation (La Rosa and Risitano, 2000; Luong, 1995). To study this relation, the average heat-dissipated energy per cycle is plotted as a function of the number of cycles to failure $N_{\mathrm{f}}$ in Figure 9. The average heat-dissipated energy per cycle is calculated for the 10,000th cycle, which corresponds to a steady state for all loading conditions. According to Figure 9, no clear correlation between heat dissipation and fatigue failure is observed. More specifically, an increase in the average dissipated energy per cycle is not necessarily associated with a reduction in the number 


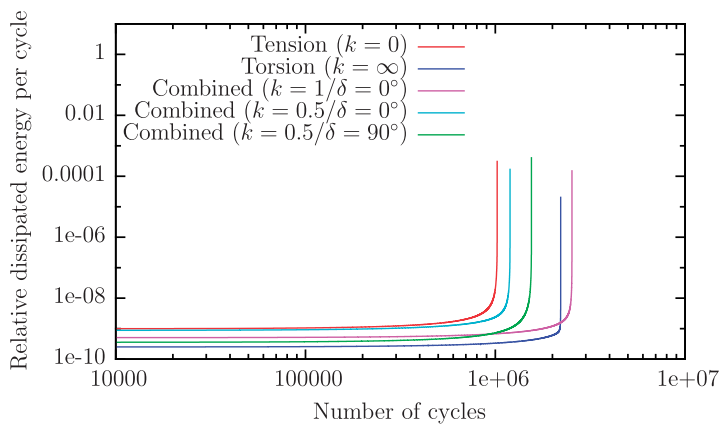

Figure 8. Evolution of the relative contribution of damage to the average heat dissipated energy per cycle for the different loading configurations.

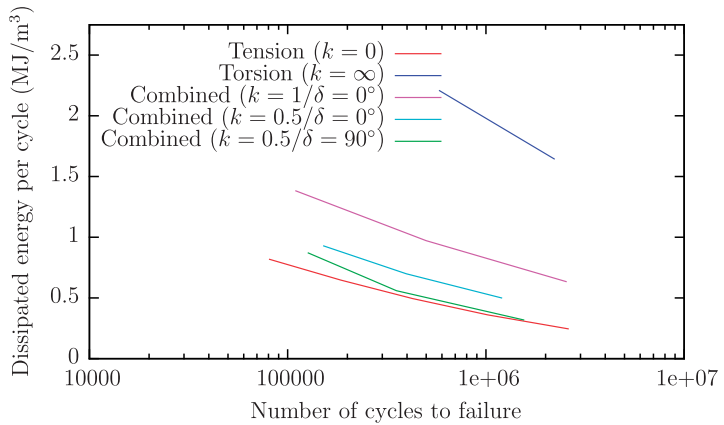

Figure 9. Average heat-dissipated energy per cycle as a function of the number of cycles to failure for the different loading configurations. The average heat dissipated energy per cycle is calculated for the 10,000th cycle.

of cycles to failure. A fatigue criterion solely based on dissipated energy would therefore appear to be inappropriate for multiaxial loading conditions.

\section{Conclusions}

In this paper, a micromechanical approach is proposed to model the high cycle fatigue behaviour of metallic materials. To account for the anisotropy of elastic and plastic single crystal properties, the constitutive model is developed at the crystal scale within the context of crystal plasticity. The introduction of a damage variable for each slip system enables description of the progressive degradation of mechanical properties. The anisotropic nature of damage is considered through the relation between the damage variables and the elastic stiffness tensor. For a given slip system, the evolution of damage is governed by both the elastic strain energy release rate and the plastic shear strain rate. A strong coupling between plasticity and damage is therefore assumed. To account for the polycrystalline nature of metallic materials, the local constitutive model is implemented within a self-consistent formulation.

The validity of the proposed model has been evaluated by studying the fatigue behaviour of a medium carbon steel (SAE 1035). The material parameters were adjusted from the experimental dataset obtained by Flacelière et al. (2007a,b), Gadouini et al. (2008) and Vu (2009). Even for 
complex loading conditions (e.g. out-of-phase combined tension-torsion), the model enables correct reproduction of the fatigue behaviour.

According to the model, independently of the loading conditions, fatigue damage is highly localized in some specific crystallites. As a result, although an important stiffness reduction is observed at the crystal scale, the macroscopic elastic properties are not significantly impacted by damage. In addition, the contribution of damage to the macroscopic dissipation source is found to be negligible in comparison with the contribution associated with plasticity. Finally, when the number of cycles to failure is compared with the heat dissipated energy for different loading conditions, no evident correlation between fatigue damage and heat dissipation is observed.

\section{Declaration of Conflicting Interests}

The authors declared no potential conflicts of interest with respect to the research, authorship, and/or publication of this article.

\section{Funding}

The author(s) disclosed receipt of the following financial support for the research, authorship, and/or publication of this article: This work is part of the APSTRAM project managed by IRT Jules Verne (French Institute in Research and Technology in Advanced Manufacturing Technologies for Composite, Metallic and Hybrid Structures). The authors wish to associate the industrial and academic partners of this project; respectively IRT Jules Verne, ENSAM Paris Tech, DCNS, STX Europe, Bureau Veritas and Europe Technologies.

\section{References}

Abdul-Latif A and Mounounga TB (2009) Damage-induced anisotropy with damage deactivation. International Journal of Damage Mechanics 18: 177-198.

Bennett VP and McDowell DL (2003) Polycrystal orientation distribution effects on microslip in high cycle fatigue. International Journal of Fatigue 25: 27-39.

Chadli M and Abdul-Latif A (2005) Mesodamage evolution in polycrystals. Journal of Engineering Materials and Technology 127: 214-221.

Cordebois JP and Sidoroff F (1979) Anisotropie élastique induite par endommagement. Euromech colloquium 115.

Crossland B (1956) Effect of large hydrostatic pressures on the torsional fatigue strength of an alloy steel. Proceedings of the International Conference on Fatigue of Metals, Institution of Mechanical Engineers, London.

Dang Van K (1973) Sur la résistance à la fatigue des métaux. Sciences et techniques de l'armement 3: 47.

Dunne FPE, Walker A and Rugg D (2007) Length scale-dependent, elastically anisotropic, physically-based HCP crystal plasticity: application to cold-dwell fatigue in Ti alloys. International Journal of Plasticity 23: 1061-1083.

Eshelby JD (1957) The determination of the elastic field of an ellipsoidal inclusion, and related problems. Proceedings of the Royal Society of London A 24: 1 376-396.

Flacelière L, Morel F and Dragon A (2007a) Coupling between mesoplasticity and damage in high-cycle fatigue. International Journal of Damage Mechanics 16: 473-509.

Flacelière L, Morel F and Dragon A (2007b) Competition between mesoplasticity and damage under HCF elasticity/damage shakedown concept. International Journal of Fatigue 19: 2281-2297.

Gadouini H, Nadot Y and Rebours C (2008) Influence of mean stress on the multiaxial fatigue behaviour of defective materials. International Journal of Fatigue 30: 1623-1633.

Huyen N, Flaceliere L and Morel F (2008) A critical plane fatigue model with coupled meso-plasticity and damage. Fatigue and Fracture of Engineering Materials and Structures 31: 12-28.

La Rosa G and Risitano A (2000) Thermographic methodology for rapid determination of the fatigue limit of materials and mechanical components. International Journal of Fatigue 22: 65-73. 
Ledbetter $\mathrm{H}$ (2010) Polycrystalline elastic constants of in situ cementite $\left(\mathrm{Fe}_{3} \mathrm{C}\right)$. Materials Science and Engineering $A$ 527: 2657-2661.

Lemaitre J (1996) A Course on Damage Mechanics, 2nd ed. Berlin: Springer-Verlag.

Lemaitre J and Chaboche J-L (1994) Mechanics of Solid Materials, 1st ed. Cambridge: Cambridge University Press.

Luong MP (1995) Infrared thermographic scanning of fatigue in metals. Nuclear Engineering and Design 158: $363-376$

Mareau C and Berbenni S (2015) An affine formulation for the self-consistent modeling of elasto-viscoplastic heterogeneous materials based on the translated field method. International Journal of Plasticity 64: $134-150$

McDowell DL (1996) Basic issues in the mechanics of high cycle metal fatigue. International Journal of Fracture 80: $103-145$.

McDowell DL (2007) Simulation-based strategies for microstructure-sensitive fatigue modeling. Materials Science and Engineering A 468-470: 4-14.

Méric L and Cailletaud G (1991) Single crystal modeling for structural calculations: Part 2 - Finite element implementation. Journal of Engineering Materials and Technology 113: 171-182.

Monchiet V, Charkaluk E and Kondo D (2006) Plasticity-damage based micromechanical modelling in high cycle fatigue. Comptes Rendus Mécanique 334: 129-136.

Mounounga TB, Abdul-Latif A and Razafindramary D (2011) Damage induced anisotropy of polycrystals under complex cyclic loadings. International Journal of Mechanical Sciences 53: 271-280.

Przybyla C, Prasannavenkatesan R, Salajegheh N, et al. (2010) Microstructure-sensitive modeling of high cycle fatigue. International Journal of Fatigue 32: 512-525.

Repetto EA and Ortiz M (1997) A micromechanical model of cyclic deformation and fatigue crack nucleation in F.C.C. single crystals. Acta Materialia 45(6): 2577-2595.

Robert C and Mareau C (2015) A comparison between different numerical methods for the modeling of polycrystalline materials with an elastic-viscoplastic behavior. Computational Materials Science 103: $134-144$

Robert C, Saintier N, Palin-Luc T, et al. (2012) Micro-mechanical modelling of high cycle fatigue behaviour of metals under multiaxial loads. Mechanics of Materials 55: 112-129.

Saanouni K and Abdul-Latif A (1996) Micromechanical modeling of low cycle fatigue under complex loadings - Part I. Theoretical formulation. International Journal of Plasticity 12: 1111-1121.

Sakata K, Daniel D and Jonas JJ (1989) Estimation of 4th and 6th order ODF coefficients from elastic properties in cold rolled steel sheets. Textures and Microstructures 11: 41-56.

Sauzay M and Gilormini P (2002) Influence of surface effects on fatigue microcracks nucleation. Theoretical and Applied Fracture Mechanics 38: 53-62.

Sweeney CA, Vorster W, Leen SB, et al. (2013) The role of elastic anisotropy, length scale and crystallographic slip in fatigue crack nucleation. Journal of the Mechanics and Physics of Solids 61: 1224-1240.

Suresh S (1998) Fatigue of Materials, 2nd ed. Cambridge: Cambridge University Press.

Vu QH (2009) Fatigue polycyclique multiaxiale de l'acier C35: caractérisation et modélisation des mécanismes d'endommagement. PhD Thesis, Ecole Nationale Supérieure de Mécanique et d'Aérotechnique. 